Bioscientia Medicina: Journal of Biomedicine \&

Translational Research

Journal Homepage: www.bioscmed.com

\title{
Anemia in Chronic Kidney Disease : Role of Hypoxia Inducible Factor Stabilizer
}

\section{Emilia $^{1^{\star}}$ Zulkhair Ali $^{2}$}

${ }^{1}$ Trainee of Nephrology and Hypertension, Internal Medicine Department, Faculty of Medicine, Universitas Sriwijaya, Palembang, Indonesia.

${ }^{2}$ Division of Nephrology and Hypertension, Internal Medicine Department, Faculty of Medicine, Universitas Sriwijaya, Palembang, Indonesia.

\section{A R T I C L E I N F O \\ Keywords: \\ Chronic Kidney Disease \\ Anemia \\ Erythropoiesis-stimulating Agents \\ Hypoxia Inducible Factor Stabilizer. \\ *Corresponding author: \\ Emilia \\ E-mail address: \\ emilia160781@gmail.com \\ All authors have reviewed and approved the final version of the manuscript.}

\begin{abstract}
A B S T R A C T
Anemia contributes to increased morbidity and mortality in chronic kidney disease patients. The pathogenesis of anemia in these patients is multifactorial, but the contribution of erythropoietin deficiency becomes greater as glomerular filtration rate declines which related to decreased nephron mass. The current standard of care includes supplemental iron, erythropoiesis-stimulating agents (ESA), and red blood cell transfusions, although each has drawbacks. Lately, concern has arisen following randomized clinical trials showing that higher hemoglobin targets and/or high ESA doses may cause significant harm including increasing cardiovascular and thrombotic events, and even death. Recent experimental and clinical studies show the promising efficacy of hypoxia inducible factor (HIF) stabilizer which stimulates endogenous erythropoietin production and enhance iron availability.
\end{abstract}

\section{https://doi.org/10.32539/bsm.v5i5.234}

\section{Introduction}

Anemia is defined by the World Health Organization as hemoglobin $(\mathrm{Hb})$ concentration of less than $13 \mathrm{~g} / \mathrm{L}$ in adult men and nonmenstruating women and less than $12 \mathrm{~g} / \mathrm{dL}$ in menstruating women. The incidence of anemia in patients with chronic kidney disease (CKD) increases as the glomerular filtration rate (GFR) declines. Anemia in CKD patients has been associated with increased risk of cardiovascular morbidity, mortality, decreased patient's quality of life and increased healthcare utilization. Population studies, including the United States National Health and Nutrition Examination Survey (NHANES) and the Prevalence of Anemia in Early Renal Insufficiency (PAERI) study, suggest that the incidence of anemia is less than 10\% in CKD stages 1 and 2, 20\% to $40 \%$ in CKD stage 3, $50 \%$ to $60 \%$ in CKD stage 4 , and more than $70 \%$ in CKD stage 5.1,2

The pathogenesis of anemia in CKD is multifactorial with inadequate production of erythropoietin (EPO) being the leading pathogenetic cause. Oher factors also contribute are erythropoiesis inhibition due to the accumulation of uremic toxins, reduced red blood cell survival, iron deficiency, malnutrition, inflammation, folate and/or vitamin B12 deficiency, dysregulated iron metabolism, oxidative stress, chronic gastrointestinal blood loss, secondary renal hyperparathyroidism, and blood losses during hemodialysis sessions in patients with end-stage renal disease (ESRD) requiring 
replacement therapy. ${ }^{3}$ The current standard of care for anemia in CKD includes erythropoiesis-stimulating agents (ESAs), suplemental iron, and red blood cell (RBC) transfusion, each of which has potential problems and variable effectiveness. ${ }^{4-6}$

Available ESAs are very effective drugs, usually obtaining significant hemoglobin $(\mathrm{Hb})$ level increases. However, recombinant ESAs are still expensive and require cold storage. In addition, parenteral and frequent administration route may be cumbersome for long-term treatment in non-dialysis-dependent CKD (NDD-CKD) patients. In dialysis dependent CKD (DDCKD) patients, the issue is more complex because it increases nurse workload. ${ }^{7}$ In addition, a series of large trials demonstrated that ESAs have serious safety problems, including increasing cardiovascular and thrombotic events that is associated with supraphysiologic ESA plasma levels, EPO-resistance caused by inflammation, hypertension, and even death. This, together with changes in ESA reimbursement policy in some countries, has led to reductions in prescribed ESA dose and target $\mathrm{Hb}$ levels. Hence, an increase in blood transfusion requirements and intravenous iron dosing has occurred. Blood transfusions cannot be considered as an alternative strategy to ESAs because they still have some risks, expose patients to large $\mathrm{Hb}$-level fluctuations, and by definition have limited availability. Moreover, they may enhance the synthesis of alloantigenic antibodies, reducing the likelihood of patients subsequently receiving a kidney transplant. Excessive iron use may cause harm as well because of its toxic effect to cells. ${ }^{4}$, $5,7-10$

Given the inherent limitations of the current standard of care, new effective and tolerable treatment options for anemia in CKD are needed. One particularly promising class of agents in development is hypoxiainducible factor-prolyl hydroxylase (HIF-PH) inhibitors also known as Hypoxia-inducible factors (HIF) stabilizer. HIF stabilizer stimulate endogenous erythropoietin (EPO) production and enhance iron availability. In early clinical trials, these oral agents appear to be capable of replacing ESAs and minimizing the need for i.v. iron therapy for anemia in CKD, while having other potentially advantageous actions. 11-14

\section{Hypoxia inducible factor system}

A number of physiological processes are relevant to normal red cell production, and the disturbance of these processes result in chronic anemia including that associated with CKD. The main processes are erythropoiesis, regulation of erythropoietin synthesis (including the role of HIF), and regulation of iron supply (including the role of hepcidin). Erythropoiesis is a tightly regulated process with the main role of EPO in terminal maturation of erythroid cells. EPO has long been recognized as the physiologic regulator of red cell production in response to hypoxia. In addition, analysis of EPO gene regulatory elements paved the way to the discovery of the HIF system. ${ }^{15}$

The hypoxia-inducible factor (HIF) system regulates the cellular transcriptional response to hypoxia. This includes the stimulation of erythropoiesis, the absorption and mobilization of iron, and the coordination of other cell-specific responses (angiogenesis, lipid and glucose metabolism, glycolysis, mitochondrial function, cell growth and survival, vasodilation, cell migration and inflammation). HIF, a heterodimer consisting of one oxygen-sensitive $\propto$ subunit and $\beta$ subunit was first identified in 1992. Notably, work on the discovery of HIF and its mechanism of action received the 2019 Nobel Prize in Physiology or Medicine. Three different $\mathrm{HIF} \propto$ isoforms exist: HIF $1 \propto, \mathrm{HIF} 2 \propto$ and HIF $3 \propto$. HIFs are controlled by a family of PHD: PHD1, PHD2 and PHD3; PHD2 is considered as the main regulator of the pathway. 4, 16, 17

The schematic presentation of HIF system depicted in figure 1. Under normoxic condition (red arrow; schematic on right side), HIF-a subunits are hydroxylated on specific proline and asparagine residues by PHDs and $\mathrm{FIH}-1$, respectively. The hydroxylation reactions utilize $\mathrm{O}_{2}$ and a-ketoglutarate as substrates and generate $\mathrm{CO}_{2}$ and succinate as byproducts. Prolyl-hydroxylated HIF-a subunits are recognized by VHL, which recruits the BCCRE2 complex composed of elongin B, elongin C, CUL2, RBX1, and an E2 ubiquitin-protein ligase, leading to 
polyubiquitination on lysine residues of the HIF-a subunits, which targets these proteins for proteasomal degradation. Hydroxylation of an asparagine residue in the transactivation domain of the HIF-a subunits blocks recruitment of the coactivators p300 and CBP. In hypoxic cells (blue arrow; schematic on left side), the hydroxylation reactions are inhibited, leading to the accumulation of HIF-a subunits, which dimerize with HIF-1 $1 \beta$, recruit coactivators, and bind to the consensus sequence 5'-(A/G)CGTG-3' in hypoxia response elements located within or near target genes, which activates their transcription. ${ }^{18-20}$ (Abbreviations: FIH1, factor-inhibiting HIF-1; HIF, hypoxia-inducible factor; PHD, prolyl hydroxylase domain protein; Ub, ubiquitin; VHL, von Hippel-Lindau protein).

\section{Hypoxia-inducible factor stabilizers}

Roxadustat (FG-4592) was the first-in-class HIF stabilizer approved in Japan for the treatment of anemia in DD-CKD and in China for patients DD-CKD or NDD-CKD. Daprodustat (GSK1278863) and vadadustat (AKB-6548) are also now approved in Japan for the treatment of anemia in patients with DD-CKD or NDD-CKD. All three HIF stabilizer stimulate EPO production in patients with anemia of CKD, providing dose-dependent increases in $\mathrm{Hb}$ and reductions in hepcidin levels, and thus improving total iron binding capacity (TIBC).4, 21 Several other HIF stabilizer are in development, with data available for molidustat (BAY 85-3934), enarodustat (JTZ-951), and desidustat (Zyan 1). 4

\section{HIF stabilizer and erythropoietin stimulation}

The physiological serum concentrations of endogenous EPO range from 4 to $27 \mathrm{mU} / \mathrm{mL}$ but increase by 100 - 1000 folds in response to hypoxia and anemia There are two different receptors for EPO; one exhibits high affinity while the other possesses low affinity for EPO. Although, the low affinity receptor mediates non-erythropoietic, tissue protective effects of EPO, it requires a high dose of exogenous EPO which creates a prothrombotic state via production of highly reactive platelets and vascular endothelium activation. In addition, higher concentrations of endogenous EPO are associated with incident heart failure in adults aged 70-79 years. This highlights the potential advantage of HIF stabilizer which lead to small incremental increases in endogenous EPO levels which are near physiological range and likely to stimulate the high affinity receptor responsible for hematopoiesis. 16, 17

Differing from ESA, HIF stabilizer correct anaemia by stimulating the production of endogenous EPO in the kidney cortex. HIF activation can also promote the hepatic production of EPO (normally occurring only during the embryonal life). Accordingly, HIF stabilizer correct anaemia in anephric patients. Following HIF stabilizer therapy, peak EPO levels are within the physiological range or well below those observed with ESA; their magnitude depends on the molecule and dose, with high inter-individual variability. 18, 19

\section{HIF stabilizer and iron metabolism}

Nearly $10 \%$ of hemodialysis population exhibits ESA resistance/hyporesponsiveness, which is often caused by iron deficiency. Although iron deficiency can be corrected with iron supplementation, the inflammatory profile of chronic kidney disease predisposes to a state of functional iron deficiency. Hepcidin, a small peptide synthesized in the liver, serves as a key culprit in the development of functional iron deficiency. Hepcidin blocks iron export and utilization via induction of degradation of the only known iron exporter, ferroportin iron. 5,8

HIF is an important regulator of iron metabolism. Because of increased erythropoiesis, hepcidin expression is reduced as an indirect effect. HIF $1 a$ can also downregulate hepcidin through Type II transmembrane serine protease matriptase-2. The lower the hepcidin level, the higher the quantity of iron available for erythropoiesis. In addition, the HIF sys tem has a direct effect on other regulators of iron transport, since it regulates the expression of the molecules involved in intestinal iron uptake (duodenal cytochrome b, apical divalent metal transporter1, ferroportin), and stimulates the synthesis of transferrin and its receptor and that of ferrochelatase (the enzyme that catalyses the insertion of iron into protoporphyrin to form haem). Iron is also a co-substrate of PHD 
enzymes, influencing their activity. Overall, HIF stabilizer improve iron availability. This could be an advantage compared with ESA, especially in patients having functional iron deficiency (little iron available for erythropoiesis despite high ferritin levels). 17

\section{HIF stabilizer and inflammation}

The HIF system plays an important role in adaptation to inflammation. HIF-1 is upregulated by bacterial and viral compounds to prepare cells of the innate and adaptive immune system to migrate to hypoxic and inflamed tissues. However, the effect of HIF stabilizer on inflammation is still an open question. Preliminary data suggest that they stimulate erythropoiesis also in inflamed patients. Indeed, dose needs with roxadustat seem to be unrelated to CRP values. Recently, a pilot study tested the efficacy of daprodustat (mean dose of $12.1 \mathrm{mg} 62.9$ day) in 15 hyporesponsive patients. At Week 16, half of the patients had suboptimal efficacy, partially because of insufficient compliance in some patients and by the choice of a too low starting dose. Interestingly, high sensitive CRP decreased during the study. ${ }^{17}$

\section{HIF stabilizer and cv risk}

The major strategy of cardiovascular risk management includes an adequate control of blood pressure, LDL cholesterol, and plasma glucose levels. In this regard, there is growing interest in the nonerythropoietic, somewhat unexpected, properties of HIF stabilizer. Several preclinical studies have suggested a potential benefit of HIF activation in myocardial infarction, cardiac remodeling, atherosclerosis, and peripheral artery disease. Ameliorating glucose and lipid metabolism and lowering blood pressure may also contribute to cardiovascular protection. 22 In phase II studies of roxadustat, total cholesterol levels were reduced significantly irrespective of the use of statins, by a mean of $26 \pm 30 \mathrm{mg} / \mathrm{dL}$ after 8 weeks of therapy. 23 Another possible CV protective effect of HIF stabilizer is the lowering of platelet count. Until now, it remains difficult to predict the overall clinical impact of HIF activation on CVD in CKD patients; the results of Phase
III trials on hard CV endpoints are awaited. ${ }^{17}$

\section{Potential disadvantages of hif stabilizer}

Although HIF stabilizers have the potential to treat renal anemia effectively, accruing evidence has shown that HIF transcription factor directly regulates hundreds of genes, and consequently plays an important role in a broad spectrum of cellular functions and biological processes other than erythropoiesis, including energy metabolism, angiogenesis, mitochondrial metabolism, cellular growth and differentiation, inflammation, cell motility, matrix production, and epigenetics. Among these undesirable effects, promoting tumor growth is the greatest concern. In the tumor microenvironment, hyperproliferative tissue is hypoxic, so that the activation of HIF is involved in tumor growth and metastasis. It is known that many tumors overexpress HIF compared with their normal tissue counterparts, and this overexpression is associated with a poor prognosis. For instance, VEGF is associated with tumor growth and metastasis by increasing the oxygen supply through the promotion of angiogenesis. It also contributes to exacerbations of diabetic retinopathy. ${ }^{19}$, 24

In addition, HIF is involved in the development of pulmonary hypertension, a condition not uncommonly comorbid with CKD. The new onset or exacerbation of pulmonary hypertension due to HIF activation is therefore a concern. FGF-23 is known to be associated with HIF-1, although clinical data on this point are currently unavailable owing to lack of long-term use of HIF stabilizer. Research addressing these potential side effects is important, but most studies thus far have involved gene knockout animal models. Such adverse events have not yet been thoroughly investigated in the context of CKD and end-stage renal disease. 19

Another potential side effect of PHD inhibitors is hepatotoxicity. A clinical trial of the compound FG 2216, which has a structure similar to that of roxadustat, was discontinued owing to a fatal case of liver necrosis, although the death was not attributed to the drug. Metabolic pathways involving glucose and fatty acids are also controlled by HIF, and a tendency 
toward elevated blood glucose levels was seen in patients taking high doses of daprodustat. Further studies including larger numbers of patients and careful longitudinal observation are necessary to determine the long-term effects of PHD inhibitors. 19, 21

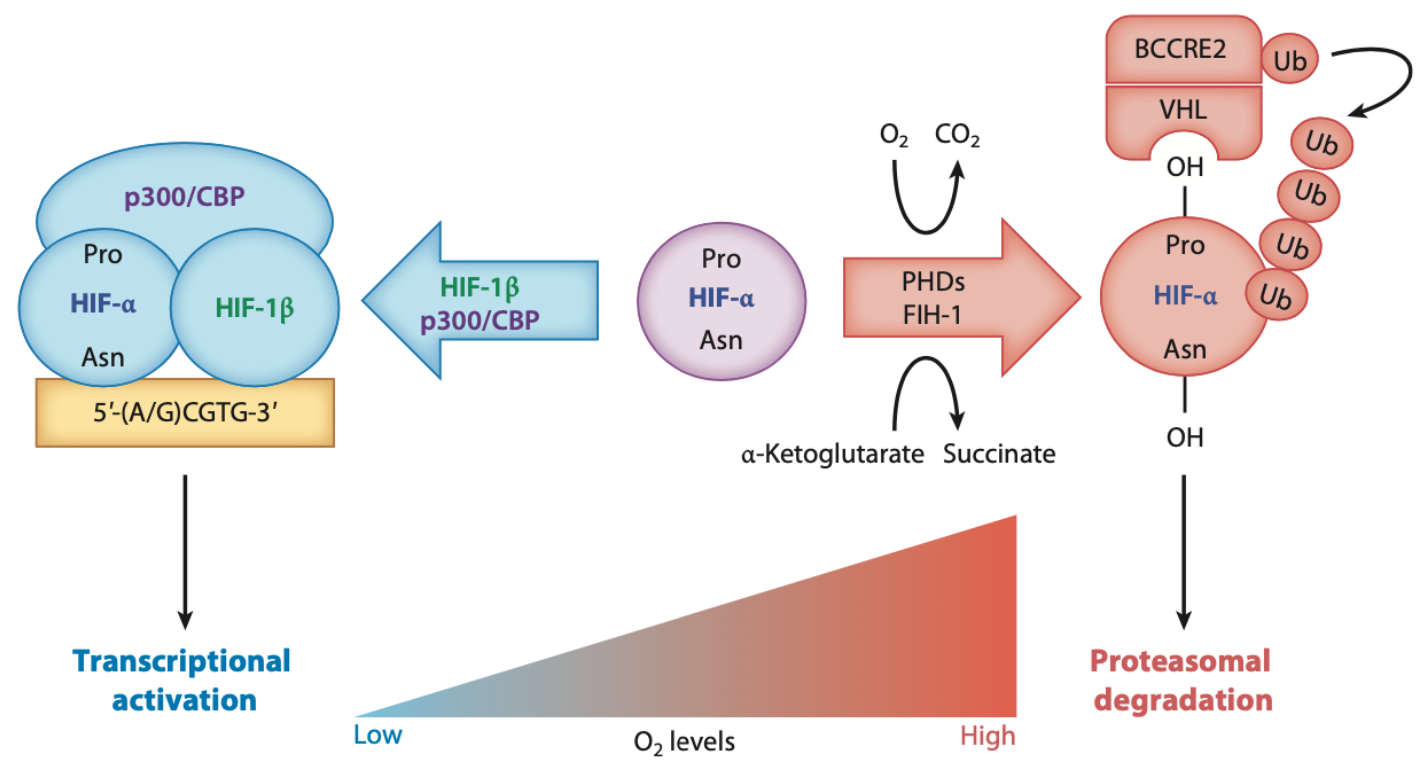

Figure 1. Oxygen dependent regulation of HIF activity ${ }^{18}$

\section{Conclusion}

The progress of anemia treatment in CKD and ESRD has liven up from very limited options in the pre-ESA era to which novel agents are developed to induce therapeutic effects that more closely resemble the body's own responses to hypoxia, raising hope that the complications caused by the prevailing ESA-based paradigms can be eliminated. The new class of orally administered HIF stabilizers that is under clinical development mimics hypoxia, resulting in the upregulation of EPO gene expression, and in an increase in both hemoglobin concentration and iron transport proteins. Because these agents may upregulate other hypoxia-sensitive genes that are involved in angiogenesis and tumor growth, their long-term safety will need to be proven.

\section{References}

1. Brugnara C, Eckardt K-U. Hematologic aspects of kidney disease. Brenner and Rector's the kidney 9th ed Philadelphia: Saunders. 2020;2:1861-99.
2. Wish JB. Anemia and Other Hematologic Complications of Chronic Kidney Disease. National kidney foundation primer on kidney diseases: Elsevier; 2018. p. 515-25.

3. Cernaro V, Coppolino G, Visconti L, Rivoli L, Lacquaniti A, Santoro D, et al. Erythropoiesis and chronic kidney disease-related anemia: From physiology to new therapeutic advancements. Med Res Rev. 2019;39(2):427-60.

4. Hanna RM, Streja E, Kalantar-Zadeh K. Burden of Anemia in Chronic Kidney Disease: Beyond Erythropoietin. Adv Ther. 2020.

5. Malyszko J, Malyszko JS, MatuszkiewiczRowinska J. Hepcidin as a therapeutic target for anemia and inflammation associated with chronic kidney disease. Expert Opin Ther Targets. 2019;23(5):407-21.

6. Group KDIGOAW. KDIGO clinical practice guideline for anemia in chronic kidney disease. Kidney Int Suppl. 2012;2(4):279-335.

7. Bonomini M, Del Vecchio L, Sirolli V, Locatelli F. New Treatment Approaches for the Anemia of CKD. Am J Kidney Dis. 2016;67(1):133-42. 
8. Macdougall IC. Iron therapy for managing anaemia in chronic kidney disease. Curr Opin Nephrol Hypertens. 2018;27(5):358-63.

9. Locatelli F, Del Vecchio L. Will there still be a role for the originator erythropoiesis-simulating agents after the biosimilars and the hypoxiainducible factor stabilizers approval? Curr Opin Nephrol Hypertens. 2018;27(5):339-44.

10. Sanghani NS, Haase VH. Hypoxia-Inducible Factor Activators in Renal Anemia: Current Clinical Experience. Adv Chronic Kidney Dis. 2019;26(4):253-66.

11. Gupta N, Wish JB. Hypoxia-Inducible Factor Prolyl Hydroxylase Inhibitors: A Potential New Treatment for Anemia in Patients With CKD. Am J Kidney Dis. 2017;69(6):815-26.

12. Haase VH. Therapeutic targeting of the HIF oxygen-sensing pathway: Lessons learned from clinical studies. Exp Cell Res. 2017;356(2):1605.

13. Kaplan JM, Sharma N, Dikdan S. HypoxiaInducible Factor and Its Role in the Management of Anemia in Chronic Kidney Disease. Int $\mathrm{J}$ Mol Sci. 2018;19(2).

14. Kular D, Macdougall IC. HIF stabilizers in the management of renal anemia: from bench to bedside to pediatrics. Pediatr Nephrol. 2019;34(3):365-78.

15. Malyszko J, Malyszko JS. Emerging drugs for the treatment of kidney disease-induced anemia. Expert Opin Emerg Drugs. 2016;21(3):315-30.

16. Yousaf F, Spinowitz B. Hypoxia-Inducible Factor Stabilizers: a New Avenue for Reducing BP While Helping Hemoglobin? Curr Hypertens Rep. 2016;18(3):23.

17. Locatelli F, Del Vecchio L. Are prolyl-hydroxylase inhibitors potential alternative treatments for anaemia in patients with chronic kidney disease? Nephrol Dial Transplant. 2020;35(6):926-32.

18. Semenza GL. Pharmacologic Targeting of Hypoxia-Inducible Factors. Annu Rev Pharmacol Toxicol. 2019;59:379-403.
19. Sakashita M, Tanaka T, Nangaku M. HypoxiaInducible Factor-Prolyl Hydroxylase Domain Inhibitors to Treat Anemia in Chronic Kidney Disease. Contrib Nephrol. 2019;198:112-23.

20. Sato K, Kumagai N, Suzuki N. Alteration of the DNA Methylation Signature of Renal Erythropoietin-Producing Cells Governs the Sensitivity to Drugs Targeting the HypoxiaResponse Pathway in Kidney Disease Progression. Front Genet. 2019;10:1134.

21. Pan SY, Chiang WC, Chen YM. The journey from erythropoietin to 2019 Nobel Prize: Focus on hypoxia-inducible factors in the kidney. $J$ Formos Med Assoc. 2021;120(1 Pt 1):60-7.

22. Tanaka T, Eckardt KU. HIF Activation Against CVD in CKD: Novel Treatment Opportunities. Semin Nephrol. 2018;38(3):267-76.

23. Provenzano R, Besarab A, Sun CH, Diamond SA, Durham JH, Cangiano JL, et al. Oral hypoxiainducible factor prolyl hydroxylase inhibitor roxadustat (FG-4592) for the treatment of anemia in patients with CKD. Clinical Journal of the American Society of Nephrology. 2016;11(6):982-91.

24. Li ZL, Tu Y, Liu BC. Treatment of Renal Anemia with Roxadustat: Advantages and Achievement. Kidney Dis (Basel). 2020;6(2):65-73. 